\title{
NUMERICAL EVALUATION OF SURFACE INTEGRALS IN THREE DIMENSIONS
}

\author{
DAVID CHIEN
}

\begin{abstract}
In this paper, we consider the evaluation of surface integrals over piecewise smooth surfaces in three dimensions. The method consists in first replacing a parametrization for the surface and the integrand function by piecewise polynomial interpolants of them, and second, using a numerical integration scheme for the resulting integral. The order of convergence is higher than would be expected based on the underlying interpolation theory.
\end{abstract}

\section{INTRODUCTION}

An efficient numerical integration scheme for surface integrals is important in boundary element methods. This paper investigates a method which consists in first replacing both the integrand function and a parametrization for the surface by piecewise polynomial interpolants of them, and then applying a numerical integration scheme to the resulting integral.

Let the surface $S=S_{1} \cup S_{2} \cup \cdots \cup S_{J}$, where each $S_{K}$ is a smooth surface. Let $F_{K}: R_{K} \stackrel{1-1}{\longrightarrow} \mathbf{R}^{3}, R_{K} \subset \mathbf{R}^{2}$, be a smooth parametrization of the surface $S_{K}$ in $\mathbf{R}^{3}$, with $D_{s} F_{K}(s, t) \times D_{t} F_{K}(s, t) \neq 0$ at all points. For each $K$, consider the evaluation of the surface integral

$$
\int_{S_{K}} f(Q) d S_{Q}=\int_{R_{K}} f\left(F_{K}(s, t)\right)\left|D_{s} F_{K}(s, t) \times D_{t} F_{K}(s, t)\right| d s d t
$$

where $f$ is a given continuous function defined on $S$, and smooth on each $S_{K}$

In this paper, we approximate the function $f$ and each parametrization $F_{K}$ by polynomials of degree $n$ and $r$, respectively. Thus, we approximate the integration (1.1) by evaluating

$$
\int_{R_{K}} f_{n}\left(F_{K}(s, t)\right)\left|D_{s} \widetilde{F}_{K}(s, t) \times D_{t} \widetilde{F}_{K}(s, t)\right| d s d t .
$$

Also, we approximate the integration (1.2) by using various numerical integration schemes.

Recently, Georg and Tausch [8] have investigated the rate of convergence for the case in which the surface parametrization is approximated by piecewise

Received by the editor August 23, 1993 and, in revised form, February 28, 1994.

1991 Mathematics Subject Classification. Primary 65D30; Secondary 65D05. 
linear interpolation. They obtained asymptotic error results when using integration rules with degree of precision zero and one. Verlinden and Cools [12] and Lyness [10] have independently proved a conjecture from Georg and Tausch [8], obtaining a full asymptotic expansion of the error.

In this paper, we refine the surface $S$ with uniform subdivision, and the results and their proofs are valid for sufficiently smooth integrand functions. Schwab and Wendland [11] carry out a detailed analysis for the singular surface integrals arising in solving boundary integral equations. Also, Yang and Atkinson [13] show how the singular case can be treated by composite quadrature with nonuniform subdivision.

We introduce the method of discretization in $\S 2$, and describe the method of interpolation for the integrand function and the surface parametrization in $\S 3$. Section 4 gives the error analysis when using (1.2), and $\S 5$ has the error analysis for the numerical integration scheme. Section 6 gives some numerical examples, which illustrate the theorem in $\S 5$.

\section{THE TRIANGULATION AND REFINEMENT}

As discussed in Atkinson [1], we assume the surface $S$ can be written as

$$
S=S_{1} \cup S_{2} \cup \cdots \cup S_{J},
$$

where each $S_{i}$ is a closed, "smooth" surface in $\mathbf{R}^{3}$. The only possible intersection of a pair $S_{i}$ and $S_{j}$ is to be along a common portion of the edges of these two subsurfaces. We assume that each $S_{i}$ has a parametrization in a region of $\mathbf{R}^{2}$, with the parametrization $r+3$ times continuously differentiable. In this case, we say $S$ is piecewise smooth. By a smooth surface, we mean that for each point $P \in S$ there is a neighborhood on $S$ of $P$ having a local $r+3$ times continuously differentiable parametrization in $\mathbf{R}^{2}$ with the Jacobian of the transformation not vanishing.

For a smooth surface $S$, we also assume that the integrand $f$ can be extended into a neighborhood of $S$, with preservation of its differentiability as a function on $S$. For $S$ piecewise smooth, as in (2.1), we assume that $f \mid s_{K}$ has a smooth extension to a neighborhood of $S_{K}$, for each $K$. The required differentiability of $f$ depends on the degree of the interpolant, i.e., if $f$ is approximated by a polynomial of degree $n$, then $f \in C^{n+2}\left(S_{K}\right)$. Thus $S_{K}$, for each $K$, should admit an at least $n+2$ times continuously differentiable parametrization $F_{K}$. For a partial discussion of the existence of extensions of $f$ preserving differentiability, see Günter [6, p. 10]

The surface $S$ of $(2.1)$ is divided into a triangular mesh

$$
\left\{\Delta_{K, N} \mid 1 \leq K \leq N\right\} \text {, }
$$

where $N$ is the total number of triangles on the surface $S$. Each $S_{j}$ is to be broken apart into a set of nonoverlapping triangularly shaped elements $\Delta_{K, N_{j}}$, about which we say more below. In referring to the element $\Delta_{K, N}$, the reference to $N$ will be omitted, but understood implicitly. Define the mesh size of (2.2) by

$$
\delta_{N}=\max _{1 \leq K \leq N} \operatorname{diam}\left(\Delta_{K}\right)
$$




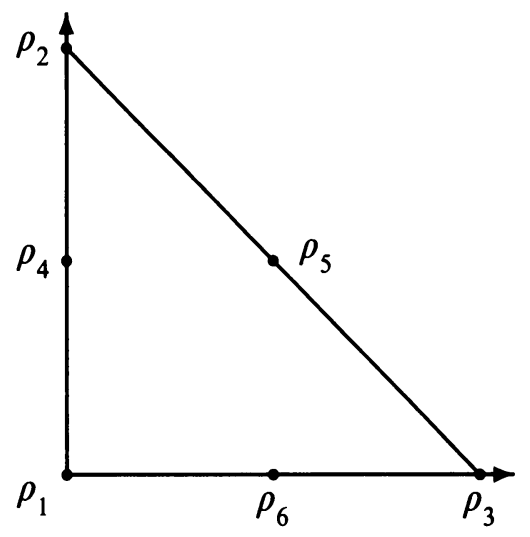

FiguRE 1. The unit simplex

$$
\operatorname{diam}\left(\Delta_{K}\right)=\max _{p, q \in \Delta_{K}}|p-q| .
$$

Let $\sigma$ denote the unit simplex in the $s t$-plane

$$
\sigma=\{(s, t) \mid 0 \leq s, t, s+t \leq 1\} .
$$

Let $\rho_{1}, \ldots, \rho_{6}$ denote the three vertices and three midpoints of the sides of $\sigma$, numbered according to Figure 1 .

One way of obtaining the triangulation (2.2) and the mappings from $\sigma$ to each $\Delta_{K}$ is by means of a parametric representation for the region $S_{j}$ of (2.1). Assume that for each $S_{j}$, there is a mapping

$$
F_{j}: R_{j} \underset{\text { onto }}{\stackrel{1-1}{\longrightarrow}} S_{j}, \quad 1 \leq j \leq J,
$$

where $R_{j}$ is a polygonal domain in the plane and $F_{j} \in C^{r+3}\left(R_{j}\right)$. Then the mapping of a triangulation of $R_{j}$, using $F_{j}$, yields a triangulation of $S_{j}$. Since the $R_{j}$ 's are polygonal domains and can be written as a union of triangles, without loss of generality, we assume in this paper that the $R_{j}$ 's are triangles. A paraboloid with top is a good example of an $S$ that satisfies our assumptions; but a circular cone is an example of an $S$ for which some of the above assumptions are not valid, because of the discontinuity of the gradient at the vertex.

Let $\widehat{\Delta}_{K}$ be an element in the triangulation of $R_{j}$, and let $\widehat{v}_{1}, \widehat{v}_{2}$, and $\widehat{v}_{3}$ be its vertices. Define

$$
m_{K}(s, t)=F_{j}\left(u \widehat{v}_{1}+t \widehat{v}_{2}+s \widehat{v}_{3}\right), \quad u=1-s-t, \quad(s, t) \in \sigma
$$

and let $\Delta_{K}$ be the image of $\widehat{\Delta}_{K}$ under this mapping. Also, if any two elements in this triangulation have a side in common, then their intersection will be an entire side of both triangles. Most surfaces $S$ of interest can be decomposed as in (2.1), with each $S_{j}$ representable as in (2.3). Also, the surface $S$ could be smooth, and we would often still want to decompose it as in (2.1). 


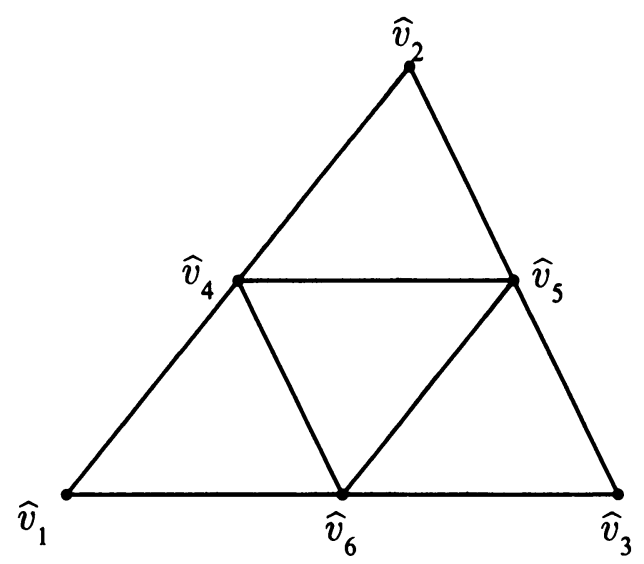

FIGURE 2. Refinement

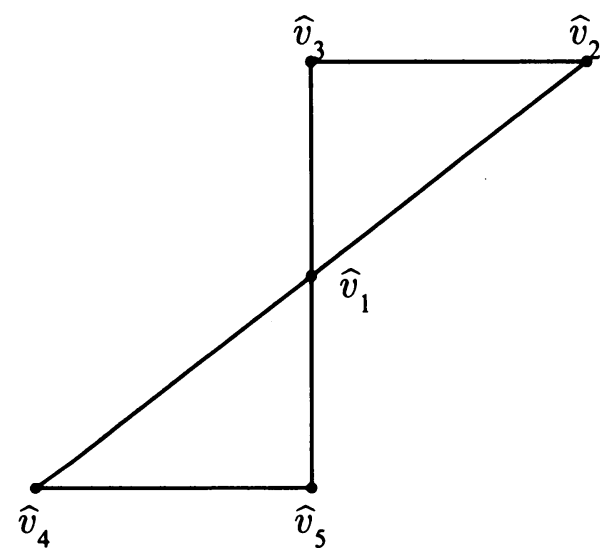

FIGURE 3. A Symmetric pair of triangles

The mapping (2.4) is used in defining interpolation and numerical integration on $\Delta_{K}$. Introduce the node points for $\Delta_{K}$ by

$$
v_{j, K}=m_{K}\left(\rho_{j}\right), \quad j=1, \ldots, 6 .
$$

The sequence of $(2.2)$ will usually be obtained by successive refinements. The refinement process is based on connecting the midpoints of the sides of a given element $\widehat{\Delta}_{K}$. Given $\left\{\widehat{v}_{1}, \ldots, \widehat{v}_{6}\right\}$, connect $\widehat{v}_{4}, \widehat{v}_{5}, \widehat{v}_{6}$ by lines parallel to the sides of $\hat{\Delta}_{K}$, as in Figure 2, producing four new triangular elements. The new elements all are congruent, and they are similar to $\widehat{\Delta}_{K}$. More importantly, in a triangulation of a polygonal region, any symmetric pair of triangles, as shown in Figure 3, has the following property:

$$
\widehat{v}_{1}-\widehat{v}_{2}=-\left(\widehat{v}_{1}-\widehat{v}_{4}\right) \text { and } \widehat{v}_{1}-\widehat{v}_{3}=-\left(\widehat{v}_{1}-\widehat{v}_{5}\right) \text {. }
$$

\section{INTERPOLATION}

We use Lagrange polynomials to define our polynomial interpolation. As an example, introduce the basis functions for quadratic interpolation on $\sigma$. 
Letting $u=1-(s+t)$, define

$$
\begin{array}{lll}
l_{1}(s, t)=u(2 u-1), & l_{2}(s, t)=t(2 t-1), & l_{3}(s, t)=s(2 s-1), \\
l_{4}(s, t)=4 t u, & l_{5}(s, t)=4 s t, & l_{6}(s, t)=4 s u .
\end{array}
$$

The functions $l_{j}(s, t)$ are quadratic Lagrange polynomials satisfying

$$
l_{i}\left(\rho_{j}\right)=\delta_{i j}
$$

Define a corresponding set of basis functions $\left\{l_{j, K}(q)\right\}$ on $\Delta_{K}$ :

$$
l_{j, K}\left(m_{K}(s, t)\right)=l_{j}(s, t), \quad 1 \leq j \leq 6, \quad 1 \leq K \leq N .
$$

Given a function $f \in C(S)$, define

$$
f_{n}(q)=\sum_{j=1}^{6} f\left(v_{j, K}\right) l_{j, K}(q), \quad q \in \Delta_{K},
$$

for $K=1, \ldots, N$. This is called the piecewise polynomial interpolation of $f$ on the nodes of the mesh $\left\{\Delta_{K}\right\}$ for $S$.

Other kinds of interpolation can be used, such as piecewise cubic interpolation in the parametrization variables, and in this case, we need ten node points, $\rho_{1}, \ldots, \rho_{10}$, and ten basis functions for the interpolation on $\sigma$. For any degree $n$, it is possible to define the needed interpolation by using $(n+1)(n+2) / 2$ uniformly spaced points on $\sigma$.

The integrand function $f\left(m_{K}(s, t)\right),(s, t) \in \sigma$, is approximated by a polynomial of degree $n$ in $(s, t)$ :

$$
f\left(m_{K}(s, t)\right) \approx f_{n}\left(m_{K}(s, t)\right) \equiv \sum_{j=1}^{n_{d}} f\left(m_{K}\left(\rho_{j}\right)\right) l_{j}(s, t),
$$

where $n_{d}$ is the number of node points required for a polynomial of degree $n$.

Because it is difficult or inconvenient to calculate the derivatives of parametrizations of many surfaces $S$, we use an approximate surface $\widetilde{S}_{N}$ with a parametrization that is easy to differentiate. The approximate surface $\widetilde{S}_{N}$ is composed of elements $\tilde{\Delta}_{1}, \ldots, \widetilde{\Delta}_{K}$, with $\widetilde{\Delta}_{K}$ an interpolant of $\Delta_{K}$. Define

$$
\tilde{m}_{K}(s, t)=\sum_{j=1}^{r_{d}} m_{K}\left(\rho_{j}\right) l_{j}(s, t)=\left[\begin{array}{c}
\sum_{j=1}^{r_{d}} v_{j, K}^{1} l_{j}(s, t) \\
\sum_{j=1}^{r_{d}} v_{j, K}^{2} l_{j}(s, t) \\
\sum_{j=1}^{r_{d}} v_{j, K}^{3} l_{j}(s, t)
\end{array}\right], \quad(s, t) \in \sigma,
$$

where $v_{j, K}^{i}$ is the $i$ th coordinate of $m_{K}\left(\rho_{j}\right)$, and $r_{d}$ is the number of node points required for a polynomial of degree $r$. Thus, $\tilde{m}_{K}(s, t)$ interpolates $m_{K}(s, t)$ at $\left\{\rho_{1}, \ldots, \rho_{r_{d}}\right\}$, and each component is a polynomial of degree $r$ in $(s, t)$.

In this paper, the interpolation methods we use for an integrand function $f$ and the surface parametrization $m$ are not necessarily the same, i.e., we 
could have two different sets of node points and two interpolants with different degrees for $f$ and $m$. Later on, we will introduce a numerical integration scheme, and we could have yet another set of node points. Thus, there might be up to three different sets of node points used in this paper, although in practice, we often have them coincide.

\section{APPROXIMATION OF INTEGRALS}

The following is the criterion for choosing node points on each $\Delta_{K}$, and it is important to the proof of Theorem 4.2.

Criterion 1. Let $g(s, t)$ be a polynomial of degree $r+1$. Let $\left\{l_{i}(s, t)\right\}$ be the Lagrange polynomials of degree $r$, and let $\left\{\rho_{j}\right\}_{j=1}^{r_{d}}$ be the associated interpolation points on $\sigma$. Let

$$
g_{r}(s, t)=\sum_{i=1}^{r_{d}} g\left(\rho_{i}\right) l_{i}(s, t)
$$

be the Lagrange form of the interpolating polynomial. Choose the node points such that

$$
\int_{\sigma} \frac{\partial}{\partial s}\left[g(s, t)-g_{r}(s, t)\right] d s d t=0
$$

and

$$
\int_{\sigma} \frac{\partial}{\partial t}\left[g(s, t)-g_{r}(s, t)\right] d s d t=0 .
$$

In this paper, using the evenly spaced node points $(i \delta, j \delta), 0 \leq i, j, i+$ $j \leq r, \delta=1 / r$, with $r$ even, satisfies the Criterion 1 , and we prove it in the following lemma.

Lemma 4.1. Using evenly spaced node points on $\sigma$ and the Lagrange form of the interpolating polynomial of degree $r$, with $r>0$ and even, satisfies Criterion 1. Proof. We have

$$
\begin{aligned}
\int_{\sigma} \frac{\partial}{\partial s} & {\left[g(s, t)-g_{r}(s, t)\right] d s d t } \\
& =\int_{0}^{1} \int_{0}^{1-t} \frac{\partial}{\partial s}\left[g(s, t)-g_{r}(s, t)\right] d s d t \\
& =\int_{0}^{1}\left\{\left[g(1-t, t)-g_{r}(1-t, t)\right]-\left[g(0, t)-g_{r}(0, t)\right]\right\} d s d t
\end{aligned}
$$

Examining (4.1), we can see that $g(0, t)-g_{r}(0, t)$ is a polynomial of degree $r+1$ in $t$. On the line segment $\{(0, t): 0 \leq t \leq 1\}$, the interpolation node points are $\{(0,0),(0,1 / r), \ldots,(0,1)\}$. Using the error formula for the interpolation error in one variable, we have

$$
\int_{0}^{1}\left[g(0, t)-g_{r}(0, t)\right] d s d t=0 .
$$


With a similar argument, we can show

$$
\int_{0}^{1}\left[g(1-t, t)-g_{r}(1-t, t)\right] d s d t=0,
$$

based on examining the interpolation error on the line segment $\{(1-t, t): 0 \leq$ $t \leq 1\}$. Therefore,

$$
\int_{\sigma} \frac{\partial}{\partial s}\left[g(s, t)-g_{r}(s, t)\right] d s d t=0
$$

and

$$
\int_{\sigma} \frac{\partial}{\partial t}\left[g(s, t)-g_{r}(s, t)\right] d s d t=0 .
$$

This completes the proof.

Criterion 1 imposes implicitly conditions for choosing node points, and Lemma 4.1 shows that evenly spaced node points satisfy the criterion. Although Lemma 4.1 did not emphasize any symmetry for the nodes, a symmetry requirement could be essential to showing Criterion 1 .

In light of Lemma 4.1 , we henceforth choose evenly spaced node points on $\sigma$, and we use the Lagrange form of the interpolating polynomial. Note that if the degree of the interpolant is $r$, the number of node points, $r_{d}$, can be calculated by the following formula:

$$
r_{d}=\frac{(r+1)(r+2)}{2}
$$

We state the first result below, in which we investigate the error of using (1.2) to approximate (1.1).

Theorem 4.2. Let $S$ be a piecewise smooth surface in $\boldsymbol{R}^{3}$. Let $\widehat{\delta}$ be the mesh size of the triangulation $\left\{\widehat{\Delta}_{K, N}\right\}$ of the various regions $R_{j}$. Use the Lagrange interpolating polynomials of degree $n$ to approximate the integrand function $f$, and use interpolation polynomials of degree $r>0$ to approximate the parametrization functions $m_{K}$. Also, choose two sets of equally spaced node points for approximating $f$ and $S$ over each triangular region. Define

$$
\tilde{r}=\left\{\begin{array}{ll}
r+2 & \text { if } r \text { is even, } \\
r+1 & \text { if } r \text { is odd },
\end{array} \quad \tilde{n}=\left\{\begin{array}{l}
n+2 \text { if } r \text { is even, } \\
n+1 \text { if } r \text { is odd }
\end{array}\right.\right.
$$

Then

$$
E_{1}=\int_{S} f(Q) d S_{Q}-\int_{\tilde{S}} f_{n}(Q) d S_{Q}=O\left(\widehat{\delta}^{u}\right),
$$

where $u=\min \{\tilde{r}, \tilde{n}\}, \tilde{S}=\tilde{S}_{1} \cup \cdots \cup \tilde{S}_{J}$, and $f \in C^{n+2}\left(S_{i}\right) \cap C(S), \quad i=$ $1, \ldots, J$.

We prove the theorem by using the following three lemmas. The first lemma examines the error of approximating the surface $S$, and the third lemma computes the error of approximating the integrand function $f$. The second lemma 
estimates an intermediate term which does not have an intuitive meaning. Write

$$
\begin{aligned}
E_{1}= & \int_{S} f(Q) d S_{Q}-\int_{\tilde{S}} f_{n}(Q) d S_{Q} \\
= & \sum_{K=1}^{N} \int_{\sigma} f\left(m_{K}(s, t)\right)\left|D_{s} m_{K} \times D_{t} m_{K}\right| d s d t \\
& -\sum_{K=1}^{N} \int_{\sigma} f_{n}\left(m_{K}(s, t)\right)\left|D_{s} \tilde{m}_{K} \times D_{t} \tilde{m}_{K}\right| d s d t
\end{aligned}
$$

with $f_{n}$ denoting the piecewise polynomial of degree $n$ interpolant of $f$. Decompose $E_{1}$ as

$$
\begin{aligned}
E_{1} & =E_{11}+E_{12}+E_{13}, \\
E_{11} & =\sum_{K=1}^{N} \int_{\sigma} f\left(m_{K}(s, t)\right)\left(\left|D_{s} m_{K} \times D_{t} m_{K}\right|-\left|D_{s} \tilde{m}_{K} \times D_{t} \tilde{m}_{K}\right|\right) d s d t, \\
E_{12}= & \sum_{K=1}^{N} \int_{\sigma}\left[f\left(m_{K}(s, t)\right)-f_{n}\left(m_{K}(s, t)\right)\right]\left(\left|D_{s} \tilde{m}_{K} \times D_{t} \tilde{m}_{K}\right|\right. \\
& \left.\quad-\left|D_{s} m_{K} \times D_{t} m_{K}\right|\right) d s d t, \\
E_{13}= & \sum_{K=1}^{N} \int_{\sigma}\left[f\left(m_{K}(s, t)\right)-f_{n}\left(m_{K}(s, t)\right)\right]\left|D_{s} m_{K} \times D_{t} m_{K}\right| d s d t .
\end{aligned}
$$

As in equation (2.4), we let

$$
m_{K}(s, t)=F_{j}\left(u \widehat{v}_{1}+t \widehat{v}_{2}+s \widehat{v}_{3}\right)=\left[\begin{array}{l}
x^{1}\left(u \widehat{v}_{1}+t \widehat{v}_{2}+s \widehat{v}_{3}\right) \\
x^{2}\left(u \widehat{v}_{1}+t \widehat{v}_{2}+s \widehat{v}_{3}\right) \\
x^{3}\left(u \widehat{v}_{1}+t \widehat{v}_{2}+s \widehat{v}_{3}\right)
\end{array}\right]
$$

for some $j$ and $u=1-s-t,(s, t) \in \sigma, x^{i} \in C^{r+3}\left(R_{j}\right), i=1,2,3$. Since the $x^{i}$ are functions of $s$ and $t$, and also of $x$ and $y$, we use both $x^{i}(s, t)$ and $x^{i}(x, y)$, with the context indicating which is intended.

Lemma 4.3. Use the Lagrange form of the interpolating polynomial of degree $r>0$ to approximate the surface parametrization $m$ as in (4.3). Define

$$
\hat{r}= \begin{cases}r+3 & \text { if } r \text { is even } \\ r+2 & \text { if } r \text { is odd }\end{cases}
$$

Then for each $\Delta_{K}$,

$$
\left|\int_{\sigma} f\left(m_{K}(s, t)\right)\left(\left|D_{s} m_{K} \times D_{t} m_{K}\right|-\left|D_{s} \tilde{m}_{K} \times D_{t} \tilde{m}_{K}\right|\right) d s d t\right| \leq C \widehat{\delta}_{K}^{\hat{r}},
$$

where $\widehat{\delta}_{K}$ is the size of $\widehat{\Delta}_{K}$, and $C$ depends on $f$ and $\left\{F_{j}\right\}$.

Proof. Let

$$
\tilde{x}^{i}(s, t)=\sum_{j=1}^{6} x^{i}\left(s_{j}, t_{j}\right) l_{j}(s, t), \quad \text { where }\left(s_{j}, t_{j}\right)=\rho_{j}, i=1,2,3 .
$$


By using the Taylor error formula, we have

$$
x^{i}(s, t)-\tilde{x}^{i}(s, t)=H^{i}(s, t)+G^{i}(s, t)+O\left(\widehat{\delta}_{K}^{r+3}\right),
$$

where

$$
\begin{gathered}
H^{i}(s, t)=\frac{1}{(r+1) !}\left[\left(s \frac{\partial}{\partial s}+t \frac{\partial}{\partial t}\right)^{r+1} x^{i}(0,0)\right. \\
\left.\quad-\sum_{j=1}^{6}\left(s_{j} \frac{\partial}{\partial s}+t_{j} \frac{\partial}{\partial t}\right)^{r+1} x^{i}(0,0) l_{j}(s, t)\right] \\
G^{i}(s, t)=\frac{1}{(r+2) !}\left[\left(s \frac{\partial}{\partial s}+t \frac{\partial}{\partial t}\right)^{r+2} x^{i}(0,0)\right. \\
\left.\quad-\sum_{j=1}^{6}\left(s_{j} \frac{\partial}{\partial s}+t_{j} \frac{\partial}{\partial t}\right)^{r+2} x^{i}(0,0) l_{j}(s, t)\right],
\end{gathered}
$$

and $O\left(\widehat{\delta}_{K}^{r+3}\right)$ comes from the $(r+3)$ rd derivative of $x^{i}(s, t)$. Note that the derivatives of $x^{i}$ with respect to $(s, t)$ give rise to formulas involving $\widehat{v}_{2}-\widehat{v}_{1}$ and $\widehat{v}_{3}-\widehat{v}_{1}$. For example,

$$
x_{s}^{i}(s, t)=\frac{\partial}{\partial s} x^{i}\left(u \widehat{v}_{1}+t \widehat{v}_{2}+s \widehat{v}_{3}\right)=\nabla x^{i} \cdot\left(\widehat{v}_{3}-\widehat{v}_{1}\right)
$$

with $\nabla x^{i}=\left[\frac{\partial x^{i}}{\partial x}, \frac{\partial x^{i}}{\partial y}\right]^{T}$. Using the Taylor error formula and expanding functions at $(s, t)=(0,0)$, we obtain

$$
\begin{aligned}
& \left|D_{s} m_{K}(s, t) \times D_{t} m_{K}(s, t)\right|-\left|D_{s} \tilde{m}_{K}(s, t) \times D_{t} \tilde{m}_{K}(s, t)\right| \\
& =E(r+2)\left(s, t ; \widehat{v}_{2}-\widehat{v}_{1}, \widehat{v}_{3}-\widehat{v}_{1}\right) \\
& \quad+E(r+3)\left(s, t ; \widehat{v}_{2}-\widehat{v}_{1}, \widehat{v}_{3}-\widehat{v}_{1}\right)+O\left(\widehat{\delta}^{r+4}\right) \\
& E(r+2)\left(s, t ; \widehat{v}_{2}-\widehat{v}_{1}, \widehat{v}_{3}-\widehat{v}_{1}\right) \\
& =\left\{\left(x_{s}^{2} x_{t}^{3}-x_{s}^{3} x_{t}^{2}\right)\left[x_{s}^{2} H_{t}^{3}+x_{t}^{3} H_{s}^{2}-x_{s}^{3} H_{t}^{2}-x_{t}^{2} H_{s}^{3}\right]\right. \\
& +\left(x_{s}^{3} x_{t}^{1}-x_{s}^{1} x_{t}^{3}\right)\left[x_{s}^{3} H_{t}^{1}+x_{t}^{1} H_{s}^{3}-x_{s}^{1} H_{t}^{3}-x_{t}^{3} H_{s}^{1}\right] \\
& \left.+\left(x_{s}^{1} x_{t}^{2}-x_{s}^{2} x_{t}^{1}\right)\left[x_{s}^{1} H_{t}^{2}+x_{t}^{2} H_{s}^{1}-x_{s}^{2} H_{t}^{1}-x_{t}^{1} H_{s}^{2}\right]\right\} /\left|D_{s} m(0,0) \times D_{t} m(0,0)\right| .
\end{aligned}
$$

Note that $x_{s}^{i}$ and $x_{t}^{i}$ are the abbreviations of $x_{s}^{i}(0,0)$ and $x_{t}^{i}(0,0)$, respectively, whereas $H_{s}^{i}$ and $H_{t}^{i}$ are functions of $(s, t)$. The error term, $E(r+2)$, is the collection of terms which are of order $r+2$ in $\widehat{\delta}$. When $r$ is even, $E(r+2)$ has the following property:

$$
E(r+2)\left(s, t ;-\left(\widehat{v}_{2}-\widehat{v}_{1}\right),-\left(\widehat{v}_{3}-\widehat{v}_{1}\right)\right)=E(r+2)\left(s, t ; \widehat{v}_{2}-\widehat{v}_{1}, \widehat{v}_{3}-\widehat{v}_{1}\right) \text {. }
$$

We do not give the explicit formula of $E(r+3)$ here, but it is the collection of terms which are of order $r+3$ in $\widehat{\delta}$. It is similar to $E(r+2)$, and it is 
an "odd function" of $\widehat{\delta}$ :

$E(r+3)\left(s, t ;-\left(\widehat{v}_{2}-\widehat{v}_{1}\right),-\left(\widehat{v}_{3}-\widehat{v}_{1}\right)\right)=-E(r+3)\left(s, t ; \widehat{v}_{2}-\widehat{v}_{1}, \widehat{v}_{3}-\widehat{v}_{1}\right)$.

Expanding $f\left(m_{K}(s, t)\right)$ about $(s, t)=(0,0)$, we have

$$
\begin{gathered}
f\left(m_{K}(s, t)\right)\left(\left|D_{s} m_{K}(s, t) \times D_{t} m_{K}(s, t)\right|-\left|D_{s} \tilde{m}_{K}(s, t) \times D_{t} \tilde{m}_{K}(s, t)\right|\right) \\
=f\left(m_{K}(0,0)\right)(E(r+2)+E(r+3)) \\
\quad+\left[s f_{s}\left(m_{K}(0,0)\right)+t f_{t}\left(m_{K}(0,0)\right)\right] E(r+2)+O\left(\widehat{\delta}^{r+4}\right) .
\end{gathered}
$$

Case 1. If $r$ is even, Lemma 4.1 shows

$$
\int_{\sigma} E(r+2)\left(s, t ; \widehat{v}_{2}-\widehat{v}_{1}, \widehat{v}_{3}-\widehat{v}_{1}\right) d s d t=0 .
$$

Therefore,

$$
\begin{aligned}
\int_{\sigma} f\left(m_{K}(s, t)\right)\left(\left|D_{s} m_{K}(s, t) \times D_{t} m_{K}(s, t)\right|\right. \\
\left.-\left|D_{s} \tilde{m}_{K}(s, t) \times D_{t} \tilde{m}_{K}(s, t)\right|\right) d s d t \\
=I E(r+3)\left(s, t ; \widehat{v}_{2}-\widehat{v}_{1}, \widehat{v}_{3}-\widehat{v}_{1}\right)+O\left(\widehat{\delta}_{K}^{r+2}\right)
\end{aligned}
$$

where

$$
\begin{aligned}
I E(r+3)(s, t & \left.; \widehat{v}_{2}-\widehat{v}_{1}, \widehat{v}_{3}-\widehat{v}_{1}\right) \\
& =\int_{\sigma}\left\{f\left(m_{K}(0,0)\right) E(r+3)\right. \\
& \left.+\left[s f_{s}\left(m_{K}(0,0)\right)+t f_{t}\left(m_{K}(0,0)\right)\right] E(r+2)\right\} d s d t
\end{aligned}
$$

Thus, this shows that

$$
\begin{aligned}
& \int_{\sigma} f\left(m_{K}(s, t)\right)\left(\left|D_{s} m_{K}(s, t) \times D_{t} m_{K}(s, t)\right|\right. \\
& \left.\quad-\left|D_{s} \tilde{m}_{K}(s, t) \times D_{t} \widetilde{m}_{K}(s, t)\right|\right) d s d t \\
& =O\left(\hat{\delta}^{r+3}\right) \quad \text { for every } \Delta_{K} .
\end{aligned}
$$

Note that

$$
\begin{aligned}
& I E(r+3)\left(s, t ;-\left(\widehat{v}_{2}-\widehat{v}_{1}\right),-\left(\widehat{v}_{3}-\widehat{v}_{1}\right)\right) \\
& \quad=-I E(r+3)\left(s, t ; \widehat{v}_{2}-\widehat{v}_{1}, \widehat{v}_{3}-\widehat{v}_{1}\right)
\end{aligned}
$$

and this odd function property will play an important role in the next lemma.

Case 2. If $r$ is odd,

$$
I E(r+2) \equiv \int_{\sigma} E(r+2)\left(s, t ; \widehat{v}_{2}-\widehat{v}_{1}, \widehat{v}_{3}-\widehat{v}_{1}\right) d s d t \neq 0,
$$

but $I E(r+2)$ has the same property as $I E(r+3)$ in (4.6). Hence, when $r$ is odd,

$\int_{\sigma} f\left(m_{K}(s, t)\right)\left(\left|D_{s} m_{K}(s, t) \times D_{t} m_{K}(s, t)\right|-\left|D_{s} \tilde{m}_{K}(s, t) \times D_{t} \tilde{m}_{K}(s, t)\right|\right) d s d t$ is $O\left(\widehat{\delta}^{r+2}\right)$ for every $\Delta_{K}$. 
Lemma 4.4. There holds $E_{11}=O\left(\widehat{\delta}^{r}\right)$.

Proof. For $r$ even, consider a symmetric pair of triangles, as in Figure 3. For the parametrizations of the two triangles, use

$$
\begin{aligned}
& m_{1}(s, t)=F\left(u \widehat{v}_{1}+t \widehat{v}_{2}+s \widehat{v}_{3}\right), \\
& m_{2}(s, t)=F\left(u \widehat{v}_{1}+t \widehat{v}_{4}+s \widehat{v}_{5}\right) .
\end{aligned}
$$

Then,

$$
\begin{aligned}
\sum_{K=1}^{2} \int_{\sigma} & f\left(m_{K}(s, t)\right)\left(\left|D_{s} m_{K}(s, t) \times D_{t} m_{K}(s, t)\right|\right. \\
& \left.\quad-\left|D_{s} \widetilde{m}_{K}(s, t) \times D_{t} \widetilde{m}_{K}(s, t)\right|\right) d s d t \\
= & I E(r+3)\left(s, t ; \widehat{v}_{2}-\widehat{v}_{1}, \widehat{v}_{3}-\widehat{v}_{1}\right)+I E(r+3)\left(s, t ; \widehat{v}_{4}-\widehat{v}_{1}, \widehat{v}_{5}-\widehat{v}_{1}\right) \\
& +O\left(\widehat{\delta}_{K}^{r+4}\right) .
\end{aligned}
$$

Using (4.6) and (2.5), we have

$$
\begin{aligned}
I E(r+3)\left(s, t ; \widehat{v}_{2}-\widehat{v}_{1}, \widehat{v}_{3}-\widehat{v}_{1}\right)+I E(r+3)\left(s, t ; \widehat{v}_{4}-\widehat{v}_{1}, \widehat{v}_{5}-\widehat{v}_{1}\right) \\
=I E(r+3)\left(s, t ; \widehat{v}_{2}-\widehat{v}_{1}, \widehat{v}_{3}-\widehat{v}_{1}\right) \\
\quad+I E(r+3)\left(s, t ;-\left(\widehat{v}_{2}-\widehat{v}_{1}\right),-\left(\widehat{v}_{3}-\widehat{v}_{1}\right)\right) \\
=0
\end{aligned}
$$

Thus, cancellation occurs on each symmetric pair of triangles, and the error contributed by each such pair of symmetric $\Delta_{K}$ is $O\left(\widehat{\delta}^{r+4}\right)$. If there are $n_{j}^{2}$ triangles for each $R_{j}$, we have $\left(n_{j}^{2}-n_{j}\right) / 2$ pairs of triangles with error of $O\left(\widehat{\delta}^{r+4}\right)$, and $n_{j}$ remaining triangles with error of $O\left(\hat{\delta}^{r+3}\right)$. We also can see that

$$
\widehat{\delta} \approx 1 / n_{j}
$$

Therefore,

$$
E_{11}=\left(n_{j}^{2}-n_{j}\right) O\left(\widehat{\delta}^{r+4}\right)+n_{j} O\left(\widehat{\delta}^{r+3}\right)=C \cdot O\left(\widehat{\delta}^{r+2}\right),
$$

i.e., the global error from using the Jacobian determinant of the approximate surface is $O\left(\widehat{\delta}^{r+2}\right)$.

If $r$ is odd, $I E(r+2)$ has the same property as $I E(r+3)$ for $r$ even, and we can use the same argument as above to get the global error of $O\left(\widehat{\delta}^{r+1}\right)$.

Lemma 4.5. Use the Lagrange form of the interpolating polynomial of degree $n$ to approximate the integrand function $f$, and use interpolation of degree $r>0$ to approximate the surface parametrization $m$. Then, $E_{12}=O\left(\widehat{\delta}^{n+r+1}\right)$. Proof. Let

$$
f\left(m_{K}(s, t)\right)-f_{n}\left(m_{K}(s, t)\right)=H_{f, K}(s, t)+O\left(\hat{\delta}^{n+2}\right),
$$


where

$$
\begin{aligned}
H_{f, K}(s, t)=\frac{1}{(n+1) !}\left[\left(s \frac{\partial}{\partial s}+\right.\right. & \left.t \frac{\partial}{\partial t}\right)^{n+1} f\left(m_{K}(0,0)\right) \\
& \left.-\sum_{j=1}^{n_{d}}\left(s_{j} \frac{\partial}{\partial s}+t_{j} \frac{\partial}{\partial t}\right)^{n+1} f\left(m_{K}(0,0)\right) l_{j}(s, t)\right] .
\end{aligned}
$$

Since

$$
f\left(m_{K}(s, t)\right)-f_{n}\left(m_{K}(s, t)\right)=O\left(\widehat{\delta}^{n+1}\right)
$$

and

$$
\left|D_{s} m_{K} \times D_{t} m_{K}\right|-\left|D_{s} \tilde{m}_{K} \times D_{t} \tilde{m}_{K}\right|=O\left(\widehat{\delta}^{r+2}\right)
$$

for every $(s, t) \in \sigma$ and for $K=1, \ldots, N$, we can conclude that

$$
\begin{aligned}
\int_{\sigma}\left[f\left(m_{K}(s, t)\right)-f_{n}\left(m_{K}(s, t)\right)\right]\left(\left|D_{s} m_{K} \times D_{t} m_{K}\right|-\left|D_{s} \tilde{m}_{K} \times D_{t} \tilde{m}_{K}\right|\right) d s d t \\
\quad=O\left(\hat{\delta}^{n+r+3}\right) .
\end{aligned}
$$

Therefore, the global error of $E_{12}$ is $O\left(\widehat{\delta}^{n+r+1}\right)$.

Lemma 4.6. There holds $E_{13}=O\left(\widehat{\delta}^{\tilde{n}}\right)$.

Proof. If $n$ is even, for every $(s, t)$ in $\sigma$ we can expand about $(s, t)=$ $(0,0)$ to obtain

$$
\begin{aligned}
\int_{\sigma}\left[f\left(m_{K}(s, t)\right)-f_{n}\left(m_{K}(s, t)\right)\right]\left|D_{s} m_{K} \times D_{t} m_{K}\right| d s d t \\
\quad=\int_{\sigma} H_{f, K}(s, t)\left|D_{s} m(0,0) \times D_{t} m(0,0)\right| d s d t+O\left(\widehat{\delta}^{n+4}\right) .
\end{aligned}
$$

Thus, we have proved that $E_{13}$ is of order $n+2$. But, if $n$ is odd, the cancellation does not occur and we can only prove that $E_{13}$ is of order $n+1$.

\section{THE NUMERICAL SCHEME}

In $\S 4$, we computed (1.1) by evaluating (1.2) analytically. Here we will evaluate (1.2) numerically and examine the error. Let $\left\{w_{j}\right\}$ represent the weights appropriate for the numerical integration scheme, and let $\left\{u_{j}\right\}$ be the node points on $\sigma$. Note that the node points $u_{j}$ are not necessarily the same as the node points we use for constructing the approximation of the integrand function $f$ and the surface parametrization $m$. Therefore, we could have three different sets of node points involved in this paper. One thing to remember is that the node points for the numerical integration scheme do not have to be evenly spaced, in contrast to the way we chose node points for interpolation in $\S 4$. 
Consider the general numerical integration scheme

$$
\begin{aligned}
\int_{S} f(Q) d S_{Q} & =\sum_{K=1}^{N} \int_{\sigma} f\left(m_{K}(s, t)\right)\left|D_{s} m_{K} \times D_{t} m_{K}\right| d s d t \\
& \approx \sum_{K=1}^{N} \sum_{j=1}^{\mu_{d}} w_{j} f_{n}\left(m_{K}\left(u_{j}\right)\right)\left|D_{s} \widetilde{m}_{K}\left(u_{j}\right) \times D_{t} \widetilde{m}_{K}\left(u_{j}\right)\right|
\end{aligned}
$$

where $\mu_{d}$ is the number of node points required for the numerical integration scheme, and $\mu$ is the degree of precision of the integration scheme, i.e., the scheme integrates exactly all polynomials of degree less than or equal to $\mu$. Using the numerical integration scheme to approximate (1.1), we have the following theorem. In order to prove the theorem, we have to assume that $f$ and $m_{K}$ for each $K=1, \ldots, J$ are at least $\mu+2$ times continuously differentiable because the numerical scheme being chosen has degree of precision $\mu$.

Theorem 5.1. Let $f, f_{n}, m$, and $\widetilde{m}$ be defined as in $\S 4$ and choose a numerical scheme which has degree of precision $\mu$. If $\mu \geq r$, then

$$
\begin{aligned}
E_{2}= & \sum_{K=1}^{N} \int_{\sigma} f\left(m_{K}(s, t)\right)\left|D_{s} m_{K} \times D_{t} m_{K}\right| d s d t \\
& -\sum_{K=1}^{N} \sum_{j=1}^{\mu_{d}} w_{j} f_{n}\left(m_{K}\left(u_{j}\right)\right)\left|D_{s} \tilde{m}_{K}\left(u_{j}\right) \times D_{t} \tilde{m}_{K}\left(u_{j}\right)\right| \\
= & O\left(\widehat{\delta}^{u}\right),
\end{aligned}
$$

where $u=\min \{\tilde{n}, \tilde{r}\}$. If $\mu<r$, then $E_{2}=O\left(\widehat{\delta}^{u}\right)$, where $u=\min \{\tilde{\mu}, \tilde{n}\}$ with $\tilde{\mu}=\mu+2$ if $\mu$ is even, and $\tilde{\mu}=\mu+1$ if $\mu$ is odd.

Proof. In order to analyze the error, we decompose the error into four parts:

$$
E_{2}=E_{21}+E_{22}+E_{23}+E_{24},
$$

where

$$
\begin{aligned}
E_{21}= & \sum_{K=1}^{N} \int_{\sigma} f\left(m_{K}(s, t)\right)\left|D_{s} m_{K} \times D_{t} m_{K}\right| d s d t \\
& -\sum_{K=1}^{N} \sum_{j=1}^{\mu_{d}} w_{j} f\left(m_{K}\left(u_{j}\right)\right)\left|D_{s} m_{K}\left(u_{j}\right) \times D_{t} m_{K}\left(u_{j}\right)\right|, \\
E_{22}= & \sum_{K=1}^{N} \sum_{j=1}^{\mu_{d}} w_{j} f\left(m_{K}\left(u_{j}\right)\right)\left(\left|D_{s} m_{K}\left(u_{j}\right) \times D_{t} m_{K}\left(u_{j}\right)\right|\right. \\
& \left.-\left|D_{s} \tilde{m}_{K}\left(u_{j}\right) \times D_{t} \tilde{m}_{K}\left(u_{j}\right)\right|\right),
\end{aligned}
$$




$$
\begin{aligned}
E_{23}= & \sum_{K=1}^{N} \sum_{j=1}^{\mu_{d}} w_{j}\left[f\left(m_{K}\left(u_{j}\right)\right)-f_{n}\left(m_{K}\left(u_{j}\right)\right)\right]\left(\left|D_{s} \tilde{m}_{K}\left(u_{j}\right) \times D_{t} \tilde{m}_{K}\left(u_{j}\right)\right|\right. \\
& \left.-\left|D_{s} m_{K}\left(u_{j}\right) \times D_{t} m_{K}\left(u_{j}\right)\right|\right) \\
E_{24}= & \sum_{K=1}^{N} \sum_{j=1}^{\mu_{d}} w_{j}\left[f\left(m_{K}\left(u_{j}\right)\right)-f_{n}\left(m_{K}\left(u_{j}\right)\right)\right]\left|D_{s} m_{K}\left(u_{j}\right) \times D_{t} m_{K}\left(u_{j}\right)\right| .
\end{aligned}
$$

$E_{21}$ is the error from the numerical integration, and $E_{22}-E_{24}$ are the errors from using the approximate surface $\tilde{m}$ and integrand $f_{n}$. For notation, recall that $f_{n}, \widetilde{w}, \widetilde{r}$, and $\tilde{n}$ are defined as in $\S 4$.

Lemma 5.2. If the numerical integration scheme being chosen has precision of degree $\mu$, then $E_{21}$ is $O\left(\widehat{\delta}^{\tilde{\mu}}\right)$.

Proof. This is a standard result and we omit the proof.

Lemma 5.3. If the degree of precision of the numerical scheme, $\mu$, is less than $r$, which is the degree of the Lagrange interpolant of the surface parametrization, then $E_{22}=O\left(\widehat{\delta}^{r}\right)$ if $r$ is even and $E_{22}=O\left(\widehat{\delta}^{r+1}\right)$ if $r$ is odd. If $\mu \geq r$, then $E_{22}=O\left(\widetilde{\delta}^{r}\right)$.

Proof. Using (4.4), we have

$$
\begin{aligned}
& f\left(m_{K}(s, t)\right)\left(\left|D_{s} m_{K}(s, t) \times D_{t} m_{K}(s, t)\right|-\left|D_{s} \tilde{m}_{K}(s, t) \times D_{t} \tilde{m}_{K}(s, t)\right|\right) \\
& =f\left(m_{K}(0,0)\right)(E(r+2)+E(r+3)) \\
& \quad+\left[s f_{s}\left(m_{K}(0,0)\right)+t f_{t}\left(m_{K}(0,0)\right)\right] E(r+2)+O\left(\widehat{\delta}^{r+4}\right)
\end{aligned}
$$

We divide the proof into two cases.

Case 1. $\mu \geq r$. If $r$ is even, (4.5) is true. Since $E(r+2)$ is a polynomial of degree $r$ in $s$ and $t$, and the degree of precision of the numerical scheme is equal or higher than $r$, we have

$$
\begin{aligned}
& \sum_{j=1}^{\mu_{d}} w_{j} E(r+2)\left(u_{j} ; \widehat{v}_{2}-\widehat{v}_{1}, \widehat{v}_{3}-\widehat{v}_{1}\right) \\
& \quad=\int_{\sigma} E(r+2)\left(s, t ; \widehat{v}_{2}-\widehat{v}_{1}, \widehat{v}_{3}-\widehat{v}_{1}\right) d s d t=0 .
\end{aligned}
$$

An argument similar to the one in the proof of Lemma 4.4 completes the proof in this case.

Case 2. $\mu<r$. In this case, (4.5) is not true, and the error contributed by each $\Delta_{K}$ is $O\left(\widehat{\delta}_{K}^{r+2}\right)$. Therefore, the global error is $O\left(\hat{\delta}^{r}\right)$ if $r$ is even, and it is $O\left(\widehat{\delta}^{r+1}\right)$ because cancellation occurs when $r$ is odd.

Lemma 5.4. There holds $E_{23}=O\left(\widehat{\delta}^{n+r+1}\right)$. 
Proof. This is a direct application of Lemma 4.5, and the degree of precision of the numerical scheme does not affect this result.

Lemma 5.5. There holds $E_{24}=O\left(\widehat{\delta}^{\tilde{n}}\right)$.

Proof. Replacing the integration in (4.7) by a numerical scheme, we get the result. Also, this result is not affected by the degree of precision of the numerical scheme which we choose to use.

Combining Lemmas 5.2-5.5 yields the assertion in Theorem 5.1.

\section{NUMERICAL EXAMPLES}

We give two sets of numerical examples by using the methods analyzed in $\S 5$. All of the numerical examples of this paper were computed on an HP 700 series work station and were computed by using the boundary element package from Atkinson [3].

The first set of numerical examples gives results for the 3-point numerical integration method. Thus, we approximate the integrand $f$ and the surface parametrization $m$ by piecewise quadratic polynomials and we approximate the integration by the formula

$$
\int_{\sigma} h(s, t) d s d t \approx \frac{1}{6} \sum_{j=4}^{6} h\left(\rho_{j}\right),
$$

which has degree of precision two over $\sigma$. This numerical scheme uses three midpoints, and all the three sets of node points are the same.

Consider the numerical evaluation of

$$
\begin{aligned}
& I=\int_{S} F(Q) d S_{Q}, \\
& F(Q)=F(x, y, z)=\frac{\partial}{\partial n_{Q}}\left(e^{z}\right) .
\end{aligned}
$$

The exterior unit normal to $S$ at $Q$ is $n_{Q}$. For $S$ we first take the ellipsoid given by

We have

$$
\frac{x^{2}}{a^{2}}+\frac{y^{2}}{b^{2}}+\frac{z^{2}}{c^{2}}=1 \text {. }
$$

$$
I=\frac{2 a b \pi}{c^{2}}\left[(c-1) e^{c}+(c+1) e^{-c}\right] .
$$

The normal derivative in the definition of $F$ is done exactly. The results are given in Table 1. The column labelled Order gives the logarithm to the base

TABLE 1. Ellipsoidal surface with $(a, b, c)=(1, .75, .5)$

\begin{tabular}{|rcc|}
\hline$N$ & Error & Order \\
\hline 8 & $2.39 \mathrm{E}-1$ & \\
32 & $3.28 \mathrm{E}-2$ & 2.87 \\
128 & $2.51 \mathrm{E}-3$ & 3.71 \\
512 & $1.66 \mathrm{E}-4$ & 3.92 \\
2048 & $1.05 \mathrm{E}-5$ & 3.98 \\
\hline
\end{tabular}


TABLE 2. Elliptical paraboloid surface with $(a, b, c)=(1, .75, .5)$

\begin{tabular}{|rcc|}
\hline$N$ & Error & Order \\
\hline 8 & $-4.29 \mathrm{E}-2$ & \\
32 & $-1.19 \mathrm{E}-2$ & 1.85 \\
128 & $-1.79 \mathrm{E}-3$ & 2.73 \\
512 & $-1.95 \mathrm{E}-4$ & 3.20 \\
2048 & $-1.80 \mathrm{E}-5$ & 3.43 \\
8192 & $-1.52 \mathrm{E}-6$ & 3.57 \\
\hline
\end{tabular}

TABLE 3. Elliptical paraboloid surface with $(a, b, c)=(1, .75, .5)$

\begin{tabular}{|rcr|}
\hline$N$ & Error & Order \\
\hline 8 & $-1.37 \mathrm{E}-1$ & \\
32 & $-1.41 \mathrm{E}-1$ & -0.04 \\
128 & $-4.66 \mathrm{E}-2$ & 1.60 \\
512 & $-1.25 \mathrm{E}-2$ & 1.90 \\
2048 & $-3.17 \mathrm{E}-3$ & 1.98 \\
\hline
\end{tabular}

two of the ratios of successive errors. Thus for $u=O r d e r$, the error at the node points is behaving like $O\left(\widehat{\delta}^{u}\right)$.

The second surface we use is an elliptical paraboloid

$$
\frac{x^{2}}{a^{2}}+\frac{y^{2}}{b^{2}}=z, \quad 0 \leq z \leq c,
$$

together with the "cap" of points $(x, y, z)$ satisfying

$$
\frac{x^{2}}{a^{2}}+\frac{y^{2}}{b^{2}} \leq c, \quad z=c .
$$

The numerical results for this surface are given in Table 2. The integral and integrand are given in (6.2), the same as for Table 1. This numerical example shows that the order of convergence approaches four more slowly than for the ellipsoidal surface.

In the next example, we use three vertices to construct the numerical scheme:

$$
\int_{\sigma} h(s, t) d s d t \approx \frac{1}{6} \sum_{j=1}^{3} h\left(\rho_{j}\right)
$$

which has degree of precision one over $\sigma$. As in the previous examples, we use piecewise quadratic approximation of the surface, but use linear interpolation for the integrand. Thus, we would expect the rate of convergence to be two, and Table 3 agrees with this. 


\section{ACKNOWLEDGMENTS}

I am grateful to K. Atkinson for helpful discussions and his interest in this problem. I am also grateful to the Mathematics Department of the University of Iowa for the use of their computers and other facilities during the preparation of this paper.

\section{BIBLIOGRAPHY}

1. K. Atkinson, Piecewise polynomial collocation for integral equations on surfaces in three dimensions, J. Integral Equations 9 (Suppl.), (1985), 25-48.

2. An empirical study of the numerical solution of integral equations on surfaces in $\boldsymbol{R}^{3}$, Reports on Computational Mathematics, \#1, Dept. of Mathematics, University of Iowa, Iowa City, IA, 1989.

3. U User's guide to a boundary element package for solving integral equations on piecewise smooth surfaces, Reports on Computational Mathematics, \#44, Dept. of Mathematics, University of Iowa, Iowa City, IA, 1993.

4. K. Atkinson and E. Venturino, Numerical evaluations of line integrals, SIAM J. Numer. Anal. 30 (1993), 881-888.

5. D. Chien, Piecewise polynomial collocation for integral equations on surfaces in three dimensions, Ph.D. thesis, Univ. of Iowa, Iowa City, IA, 1991.

6. N. Günter, Potential theory and its applications to basic problems of mathematical physics, Ungar, New York, 1967.

7. A. Stroud, Approximate calculation of multiple integrals, Prentice-Hall, Englewood Cliffs, NJ, 1971.

8. K. Georg and J. Tausch, Some error estimates for the numerical approximation of surface integrals, Math. Comp. 62 (1994), 755-763.

9. J. Lyness, Quadrature over a simplex, SIAM J. Numer. Anal. 15 (1978), 122-133 and 870887.

10. _ Quadrature over curved surfaces by extrapolation, Math. Comp. 63 (1994), 727-740.

11. C. Schwab and W. Wendland, On numerical cubatures of singular surface integrals in boundary element methods, Numer. Math. 62 (1992), 343-369.

12. P. Verlinden and R. Cools, Proof of a conjectured asymptotic expansion for the approximation of surface integrals, Math. Comp. 63 (1994), 717-725.

13. Y. Yang and K. Atkinson, Numerical integration for multivariable functions with point singularities, Reports on Computational Mathematics, \#41, Dept of Mathematics, University of Iowa, Iowa City, IA, 1993.

Department of Mathematics, California State University, San Marcos, San Marcos, CALIFORNIA 92096-0001

E-mail address: chiencabel.csusm.edu 